\title{
New Uranian satellite belt
}

\section{from David $W$. Hughes}

ON 10 March at about 21.00 universal time the planet Uranus passed across the star SAO 158687 in the constellation of Libra. Reports of this occultation have caused considerable excitement because not only was the star occulted by the planet but it was also occulted by a series of much smaller satellite objects that seem to be in an equatorial belt around the planet.

The occultation was first predicted by Gordon Taylor of the Royal Greenwich Observatory (J. Br. astr. Assoc. 83, 352; 1973). No useful observations of an Uranian occulation had been made before and even for this one the occultation would only be visible from a small area of the Earth. Also Uranus would be about 40 times brighter than SAO 158687 which has a photographic magnitude of +10 , so Taylor predicted that the observations would be extremely difficult and would need complex photoelectric equipment.

Occultation observations are important because they can provide data as to the size of the planet, its limb darkening and the extent and composition of its atmosphere. They also provide an accurate spot measurement of the planet's position which is extremely important when it comes to using celestial mechanics to calculate planetary perturbations.

The positions of Uranus, given in the Astronomical Ephemeris, and of the star SAO 158687, given in the Smithsonian catalogue, were carefully checked in January 1977 using photographic plates obtained with the $155-\mathrm{cm}$ astrometric reflector at Flagstaff, Arizona. Franz, Wasserman, Seidelmann, Van Flandern and Marsden (IAU Circ. 3038/40) reported that the position of the star had to be corrected by +1.15 arc $s$ in declination. Transit circle observations of Uranus indicated that its declination should also be corrected by -0.22 arc $s$. This meant that the occultation would not be visible everywhere that Uranus was above the horizon, but would only be seen in an area whose northern limit extended from equatorial Africa, across the Indian Ocean, somewhat north of Perth, Western Australia and then into daylight. There was an uncertainty of about $\pm 2,500 \mathrm{~km}$ in this northern limit caused by the uncertainty in the positions of the planet and star and an additional uncertainty of about $1,500 \mathrm{~km}$ due to the fact that the exact radius of Uranus is not known. Occultation would occur near $21 \mathrm{~h} 00 \mathrm{~min}$ UT and the duration would increase as one moved south.

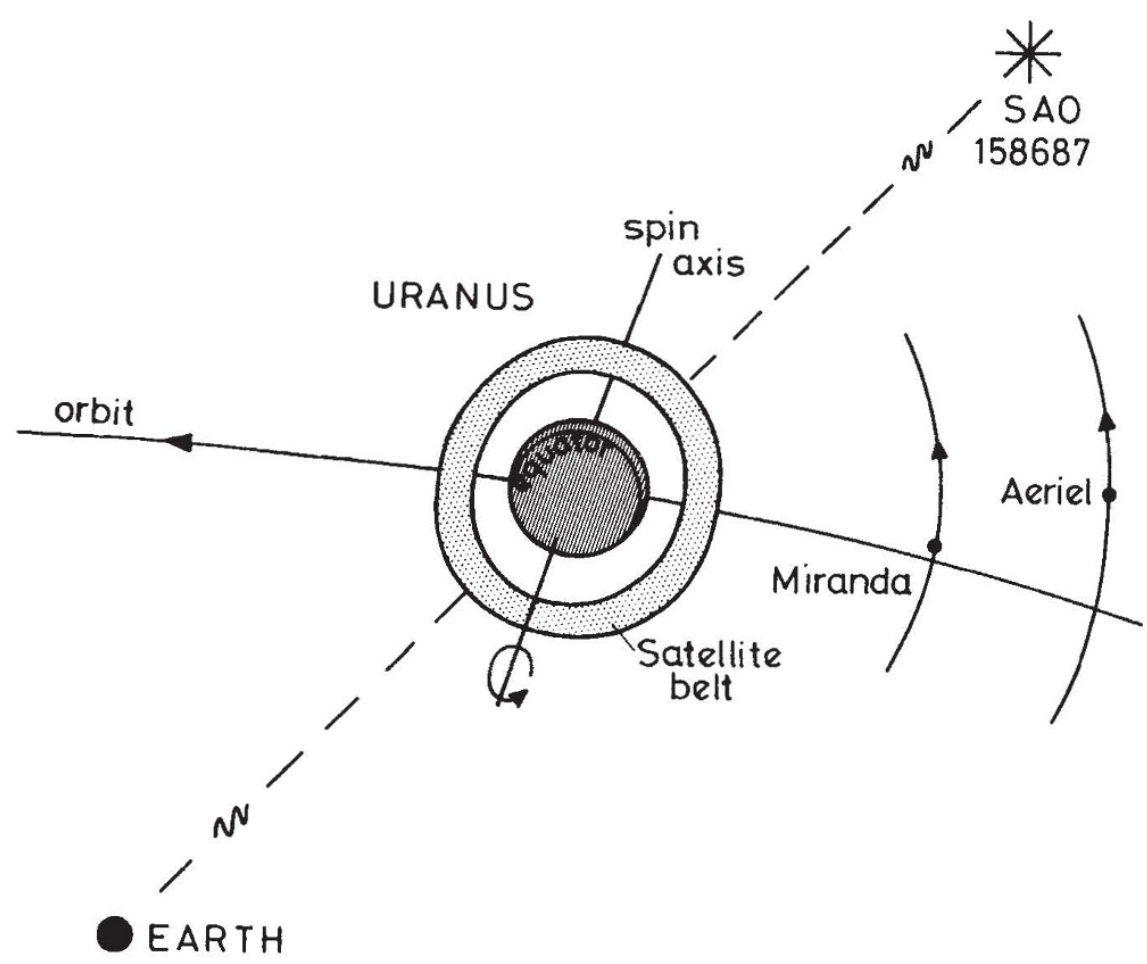

Fig. 1 A schematic diagram of Uranus and its newly discovered satellite belts at mid-occultation on 10 March, 1977. Miranda and Aeriel are the two innermost satellites, SAO 158687, the occulted star, is a 10th magnitude, K5 spectral type star in Libra. The equator of Uranus, the satellite belt and the orbital planes of Aeriel, Umbriel, Titania, Oberon and Miranda are all inclined at about $98^{\circ}$ to the ecliptic plane of the Solar System.

On 10 March heavy rain prevented observations in the vicinity of Johannesburg; however astronomers on board the Gerald P. Kuiper Airborne Observatory flying across the southern Indian Ocean about 1,000 miles East of Kerguelen Island (long. 90E, lat. 50S) were more fortunate. The occultation lasted for about $25 \mathrm{~min}$ around $21 \mathrm{~h} 06 \mathrm{~min}$ ur. Dunham, Elliot and Mink, the Cornell University astronomers on the plane also, to their surprise, saw a series of secondary occultations that took place during an $8-9 \mathrm{~min}$ interval around $20 \mathrm{~h} 16 \mathrm{~min}$ uT and during a similar interval around $21 \mathrm{~h} 50 \mathrm{~min}$. Bhattacharyya and Kuppuswamy (Indian Institute of Astrophysics) using the 102-cm reflector at Kavalur, Madras saw the secondary occultation start at $20 \mathrm{~h}$ $19 \mathrm{~min} 51 \mathrm{~s}$ and last for $8-9 \mathrm{~s}$ (they also observed that the star faded by 0.046 magnitude when it went behind the atmosphere of Uranus). Millis, Birch and Trout at the Perth Observatory, Western Australia saw the earlier of the two secondary occultations. All the groups independently concluded that these occultations were caused by satellites that are apparently part of a belt about $48,000 \mathrm{~km}$ from the centre of Uranus. This belt is shown schematically in Fig. 1. As Uranus travels along its orbit the left hand portion of the belt occults the star first then the planet occultation occurs. Finally the right hand belt occults.

Brian Marsden (Smithsonian Astrophysical Observatory) has used the times of occultation to compute the following details (IAU Circ. 3048, $3051)$. The belt is circular, in the plane of Uranus' equator, about $12,000 \mathrm{~km}$ wide and extends between 42,200 and $54,300 \mathrm{~km}$ from the centre of Uranus.

The fact that the times of the individual secondary occultations are remarkably symmetrical with respect to the Uranian occultation suggests that the occulting material is confined to specific narrow rings in the belt. The Kavalur observation seems to have been caused by a $100 \mathrm{~km}$ sized satellite near the outer edge of the belt.

Elliot, of the Centre for Radiophysics and Space Research, Cornell University, is continuing the detailed study of the occultation records. It might also be possible to observe the belts directly, although a very large telescope will be required as the brightest satellite is expected to have a magnitude of about 19. At the present opposition of Uranus the belts are between 3.5 and 40 arcs to the north and south of the planet and 2.7 to $3.1 \mathrm{arcs}$ to the east and west. 